\title{
An addition to the knowledge of lichenicolous fungi of Greece with a key to the lichenicolous fungi on Collema s.l.
}

\author{
Wolfgang von Brackel ${ }^{1} \&$ Peter Döbbeler $^{2}$ \\ ${ }^{1}$ Kirchenweg 2, 91341 Röttenbach, Germany. E-mail: wolfgang@vonbrackel.de \\ ${ }^{2}$ Ludwig-Maximilians-Universität München, Systematische Botanik und Mykologie, \\ Menzinger Str. 67, 80638 München, Germany.
}

\begin{abstract}
Didymellopsis pulposi, Pronectria diplococca, P. pedemontana, Stigmidium hageniae and Tremella anaptychiae are reported as new to Greece. Pronectria diplococca is reported as new to Austria. A key to the lichenicolous fungi on Collema s.l. is provided.
\end{abstract}

Keywords: lichen-inhabiting fungi, Mediterranean area, Bionectriaceae

\section{INTRODUCTION}

The knowledge about lichenicolous fungi of Greece was summarized in Abbott (2009), who listed 72 species known from the country. Together with four species from obviously overlooked papers (Calatayud et al., 2002; NavarroRosinés \& Hladún, 1990; Orange, 1990; Roux et al., 1995) and ten species reported after the publication of aforementioned survey (Calatayud et al., 2013; Christensen \& Alstrup, 2013; Fleischhacker et al., 2016; Hafellner, 2009; Sipman \& Raus, 2015), 86 taxa of lichenicolous fungi were known from Greece until now.

In 1971 and 1981 the second author collected microfungi on bryophytes in Greece, and took by chance also some fungi living on lichens, which were subsequently identified by the first author.

\section{MATERIAL AND METHODS}

The specimens were studied macroscopically with a Zeiss stereo microscope at magnifications up to $\times 40$ and microscopically with an Olympus BX 51 microscope fitted with Normarski differential interference contrast optics up to $\times 1000$. Measurements were taken from thin hand-cut razor-blade sections mounted in water and indicated as (minimum-) $\mathrm{X}-\sigma_{\mathrm{x}}-\mathrm{X}+\sigma_{\mathrm{x}}(-$ maximum), followed by the number of measurements (n) when $\mathrm{n} \geq 10$; the length/breadth ratio of ascospores is indicated as $1 / \mathrm{b}$ and given in the same way. For testing chemical reactions and staining the standard reagents, $10 \% \mathrm{KOH}(\mathrm{K})$, Lugol's iodine, directly or after $10 \% \mathrm{KOH}$ pre-treatment, and phloxin were used. Specimens are kept in the private herbarium of W. v. Brackel (hb Brackel). Species new to Greece are denoted with an asterisk $\left(^{*}\right)$ in the list below.

\section{RESULTS}

\section{The species}

Catillaria mediterRanea Hafellner - Greece, Attiki, Párnis $\mathrm{N}$ of Athen, above Agia Trias, $38^{\circ} 10^{\prime}$ $24^{\prime \prime} \mathrm{N}, 23^{\circ} 43^{\prime} 30^{\prime \prime} \mathrm{E}$, on bark of a coniferous tree, on Anaptychia ciliaris, 24.09.1981, leg. G. \& P. Döbbeler (hb Brackel 8411, 8418). - This is a lichenized species often growing on Anaptychia ciliaris but reported also from Niebla bourgeana, Parmelina tiliacea, Physcia semipinnata, P. stellaris, Seirophora villosa and Squamarina sp. It is known from several countries around the Mediterranean Basin including Greece, and from the Canary Islands.

*Didymellopsis pulposi (Zopf) Grube \& Hafellner - Greece, Attiki, Párnis N of Athen, Abies cephalonica forest near Agia Trias, $38^{\circ} 10^{\prime}$ $24^{\prime \prime} \mathrm{N}, 23^{\circ} 43^{\prime} 30^{\prime} \mathrm{E}$, on rocks, on Collema sp., 1100-1200 m, 11.04.1971, leg. P. Döbbeler (hb Brackel 8414, Fig. 1B). - This is a species confined to host lichens of the family Collemataceae, widespread in Europe and known also form Asia and both Americas (Brackel, 2014).

Didymellopsis sp. - Greece, Fokís, Abies forest W of Pass Amvléma, S of Graviá, near the road between Lamía and Ámfissa, 38 $37^{\prime} 10^{\prime \prime} \mathrm{N}$, $22^{\circ} 22^{\prime} 38^{\prime \prime} \mathrm{E}$, on limestone rock, on Collema auriforme, 900 m, 19.09.1981, leg. G. \& P. Döb- 

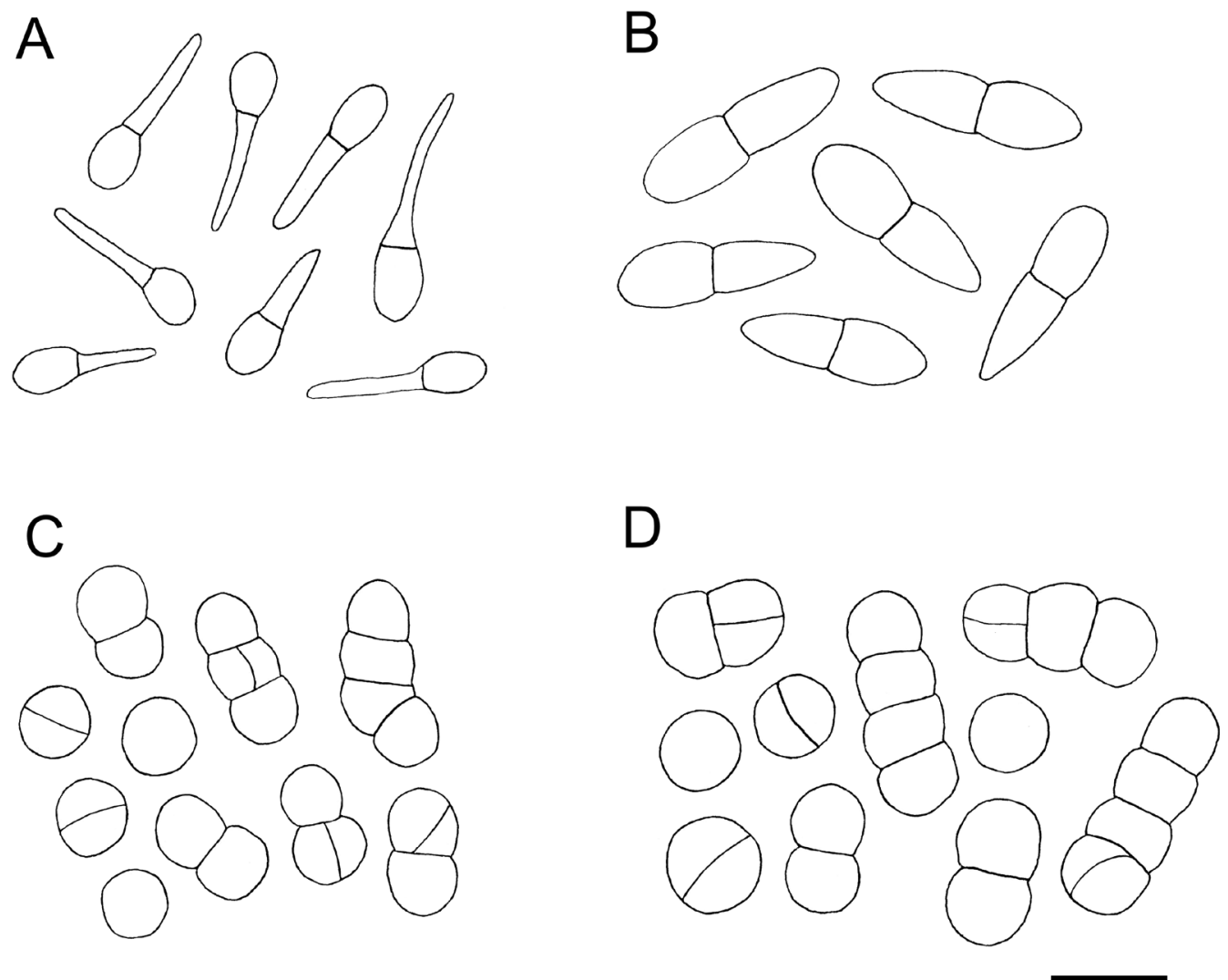

Fig. 1. Outlines of ascospores: A. Didymellopsis sp. (hb Brackel 8412); B. Didymellopsis pulposi (hb Brackel 8414); C. Pronectria diplococca from Greece (hb Brackel 8419); D. Pronectria diplococca from Austria (hb Brackel 8420). Scale bar $=10 \mu \mathrm{m}$.

beler (hb Brackel 8412, Fig. 1A). - The examined specimen fits the features of Didymellopsis pulposi, except for the shape of the ascospores, with the upper cell ellipsoid to almost orbicular, the lower one long and extremely narrow, giving the spore a caudate appearance; they measure $(12.5-) 14.4-18.6(-20.0) \times(4.0-) 4.1-4.8(-5.0)$ $\mu \mathrm{m}, 1 / \mathrm{b}=(2.6-) 3.2-4.2(-4.5)(\mathrm{n}=20)$. None of the described species of the genus has such ascospores. Caudate 1-septate ascospores are known from some species in the genus Cercidospora. The well delimited brown wall of the apothecia in our find shows that it does not belong to Cercidospora, where the mostly bluish or greenish pigmented wall is not well delimited from the host tissue. Further finds must prove if the specimen belongs to an undescribed species of Didymellopsis or represents an aberrant morph of D. pulposi.
*PRONECTRIA DIPLOCOCCA Kocourk., Khodos., Naumovich, Vondrák \& Motiej. - Greece, Pelopónnisos, Achaía, E of Patras, Abies forest c. $9 \mathrm{~km}$ $\mathrm{S}$ of Ano Diakoptón near the road Kaláyrita in direction to Diakoptón at the coast, $38^{\circ} 04^{\prime} 38^{\prime \prime} \mathrm{N}$, $22^{\circ} 10^{\prime} 04^{\prime \prime E}$, on Collema sp., 02.10.1981, leg. G. \& P. Döbbeler (hb Brackel 8419, Fig. 1C). - A very characteristic species due to its initially 1 -septate ascospores, (9.0-)9.5-10.5(-11.0) $\times(6.0-) 6.2-6.9(-7.0) \mu \mathrm{m}, 1 / \mathrm{b}=1.4-1.6(-1.7)$ ( $\mathrm{n}=20$; only 1 -septate ascospores measured) $[(8.75-) 9.56-12.34(-13.5) \times(4.75-) 5.76-7.56(-$ $8.75) \mu \mathrm{m}(\mathrm{n}=30)$ according to Khodosovtsev et al., 2012], strongly constricted at the septum, easily disintegrating. Both the 2-celled ascospores and the one-celled spore parts often develop secondary septa, mostly perpendicular to the primary septum or oblique, rarely parallel. This results in a mixture of 1-6-celled 
ascospores, some of them submuriform. If they aggregate secondarily as mentioned in the original description is not quite clear to us. The species was known until now from the Czech Republic, Germany, Lithuania and Ukraine (Khodosovtsev et al., 2012; Wagner \& Schacherer, 2019). We report this species also as new for Austria: Salzburg, Lungau, Radstädter Tauern, W Mauterndorf, slopes between Speiereck and K1. Lanschütz, on Collema cf. auriforme, MTB 8847, 2300 m, 07.09.1981, leg. P. Döbbeler (hb Brackel 8420, Fig. 1D).

*Pronectria pedemontana Brackel - Greece, Pelopónnisos, Táigetos, road from Sparta to Kalamáta, c. $2 \mathrm{~km}$ E of Artemisia, $37^{\circ} 04^{\prime} 52^{\prime \prime} \mathrm{N}, 22^{\circ} 14^{\prime} 31^{\prime \prime} \mathrm{E}$, wet place, on Collema sp., 28.09.1981, leg. G. \& P. Döbbeler (hb Brackel 8413). - The species, confined to the host genus Collema, was known until now only from Germany and Italy (Brackel, 2013).

*STigmidium Hageniae (Rehm) Hafellner - Greece, Attiki, Párnis $\mathrm{N}$ of Athen, above Agia Trias, $38^{\circ} 10^{\prime} 24^{\prime \prime} \mathrm{N}, 23^{\circ} 43^{\prime} 30^{\prime \prime} \mathrm{E}$, on bark, on Anaptychia ciliaris, 24.09.1981, leg. G. \& P. Döbbeler (hb Brackel 8416). - Ascomata 50-70 $\mu \mathrm{m}$ in diameter; asci 8-spored, 35-42 $\times 12-14$ $\mu \mathrm{m}$; ascospores hyaline, 2-celled, soleiform,

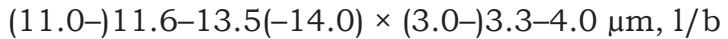
$=(2.8-) 3.0-4.0(-4.7)(\mathrm{n}=10)$. This is in accordance with the measurements given by Winter (1872): ascomata 36-100 $\mu \mathrm{m}$ diam., asci 29-43 $\mu \mathrm{m}$ long, ascospores 8-14 × 3-4 $\mu \mathrm{m}$. Vondrák et al. (2008) reported Stigmidium aff. hageniae from Crete with bigger ascospores, 13-16 $\times 5 \mu \mathrm{m}$.

*Tremella anaptychiae J. C. Zamora \& Diederich - Greece, Attiki, Párnis $\mathrm{N}$ of Athen, above Agia Trias, $38^{\circ} 10^{\prime} 24^{\prime \prime} \mathrm{N}, 23^{\circ} 43^{\prime} 30^{\prime \prime} \mathrm{E}$, on bark, on Anaptychia ciliaris, 24.09.1981, leg. G. \& P. Döbbeler (hb Brackel 8417). - This recently described species was previously known from Italy (mainland and Sardinia), Macedonia, Spain (mainland and Canary Islands) and Sweden (Zamora et al., 2017; Brackel \& Berger, 2019).

Identifying the above-mentioned fungi on lichens of the genus Collema we found that literature regarding these fungi is very scattered and the available keys to lichenicolous fungi are not very helpful, as several of the species have been described recently. Thus we present the following key, based on information from Aptroot et al. (1997), Brackel (2013, 2014), Brackel \& Etayo (2010), Clauzade et al. (1989), David \& Etayo (1995), Diederich \& Puntillo (1995), Etayo (2001, 2002, 2010), Etayo \& Sancho (2008), Grube \& Hafellner (1990), Hawksworth (1980, 1981), Hoffmann \& Hafellner (2000), Keissler (1930), Khodosovtsev et al. (2012), López de Silanes et al. (2009), Navarro-Rosinés et al. (1999), Nylander (1873), Rossman et al. (1999), Roux \& Triebel (1994), Timdal (1991), Vouaux (1913, 1914) and Zhurbenko (2009).

\section{Key to the lichenicolous fungi growing on Collema s.1.}

1 Spores produced in asci. . . . . . . . . . . . . . . . . . . . . 2

$1^{\prime}$ Spores produced in pycnidia, pycnidia setose, conidia $5-6 \times 2 \mu \mathrm{m} \ldots \ldots \ldots \ldots$

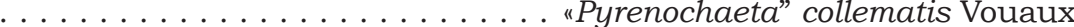

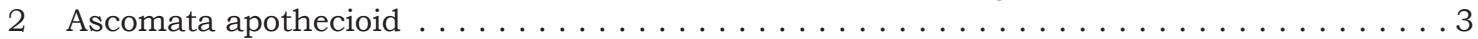

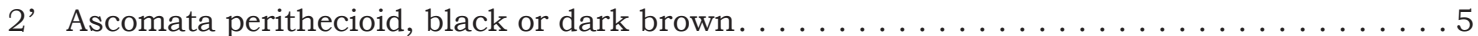

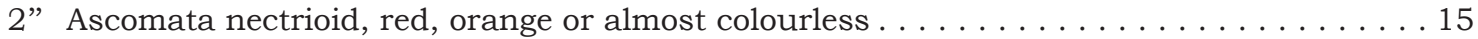

3 Apothecial disc grey, margin whitish, ascospores aseptate . . . . . "Mollisia" collematis Boud.

3' Apothecial disc and margin black, ascospores septate . . . . . . . . . . . . . . (Toninia) 4

4 Ascospores 3(-5)-septate, bacilliform to acicular . . . . . . . . Toninia collematicola Timdal

4' Ascospores 1-septate, narrowly fusiform to bacilliform. . . . . . . . . . Toninia leptogii Timdal

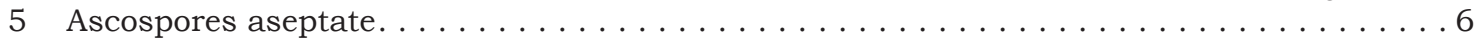

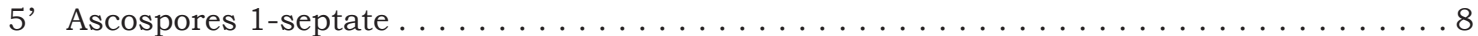

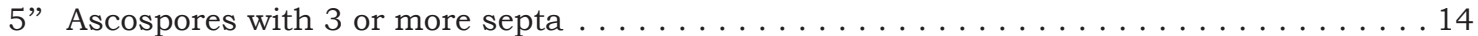

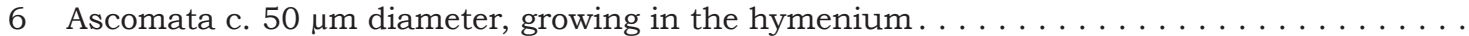
. . . . . . . . . . . . . . . . . . Physalospora collematis (Stein) G. Winter

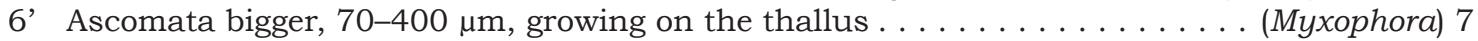

7 Asci 4-spored, ascospores (11-)14.5-19.2(-20) × (4.0-)4.7-6.9(-7.0) $\mu \mathrm{m} \ldots \ldots \ldots \ldots$. . . . . . . . . . . . . . . . . . . . . . . Myxophora tetraspora Nik. Hoffm. \& Hafellner 
7' Asci 8-spored, ascospores (10-)10.7-20.6(-23.0) × (3.0-)3.9-6.4(-7.5) $\mu \mathrm{m} \ldots \ldots \ldots \ldots . .$. . . . . . . . . . . . Myxophora leptogiophila (Minks ex G. Winter) Nik. Hoffm. \& Hafellner

7" Asci 8-spored, ascospores (9.0-)10.4-13.7(-14.0) × (6.0-)7.0-8.2(-9.0) $\mu \mathrm{m} \ldots \ldots . . . . .$. . . . . . . . . . . . . . . . . . Myxophora ovalispora Nik. Hoffm. \& Hafellner

8 Ascospores brown, hamathecial elements soon gelatinising . . . . . . . . . . (Endococcus) 9

8' Ascospores hyaline, hamathecial elements mostly persistent . . . . . . . . . . . . . 10

9 Ascospores caudate, with a tail-like lower cell, $13-21 \times(3.5-) 4.0-5.5 \mu \mathrm{m} \ldots \ldots \ldots \ldots \ldots .$. . . . . . . . . . . . . . . . . . . Endococcus caudisporus J. C. David \& Etayo

9' Ascospores heteropolar, not caudate, ascospores $9-18 \times 5-7 \mu \mathrm{m} \ldots \ldots \ldots \ldots \ldots$. . . . . .... . . Endococcus pseudocarpus Nyl. (incl. E. pellax Nyl. and "Tichothecium" latzelii Keissl.)

10 Ascomata carbonised, opening with radial ruptures . . . . . . . . . . . . . . . . . . . . . . Rhagadostoma collematum Etayo \& Nav.-Ros.

10' Ascomata not carbonised, opening with a regular ostiole $\ldots \ldots \ldots \ldots \ldots \ldots \ldots$

11 Infection gall-inducing, ascomata with numerous lipid droplets . . . . . . . . . . . . . . . . . . . . . . Lichenochora collematum Nik. Hoffm. \& Hafellner

11' Infection not gall-inducing, ascomata without numerous lipid droplets . . (Didymellopsis) 12

12 Ascospores 20-26 × 5-19 $\mu \mathrm{m}$. . . . . Didymellopsis collematum (J. Steiner) Grube \& Hafellner

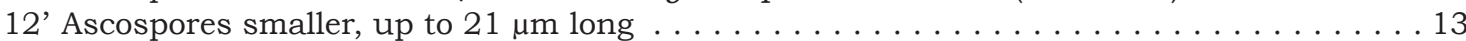

13 Ascospores heteropolar, not caudate, $14-21 \times 5-7 \mu \mathrm{m} \ldots \ldots \ldots \ldots \ldots \ldots$ . . . . . . . . . . . . . . . . Didymellopsis pulposi (Zopf) Grube \& Hafellner

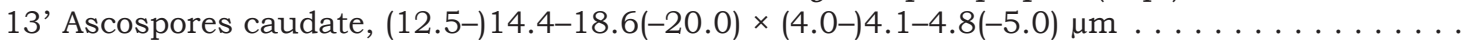

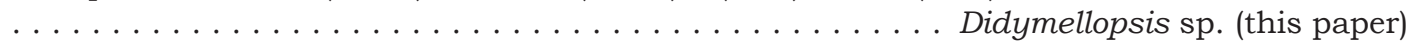

14 Ascospores parallel 3-septate, dark brown, apically paler. . . . . . Pyrenidium actinellum Nyl.

14' Ascospores parallel 3-5-septate, hyaline . . . . . . . . Sphaerulina dolichotera (Nyl.) Vouaux

14" Ascospores submuriform, reddish brown . . . . . . . . . . Pleospora collematum Zukal

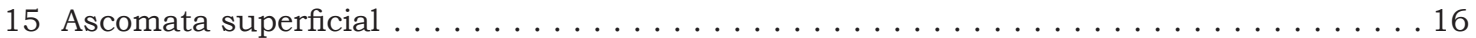

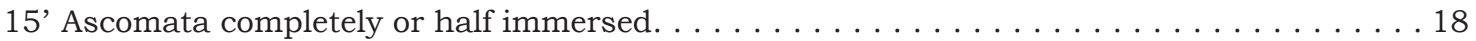

16 Ascomata without setae or hairs . . . . . . . . . "Nectria" brutia Diederich \& Puntillo

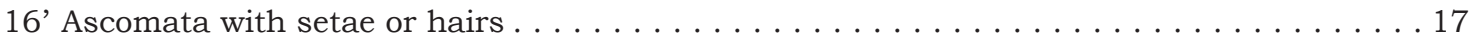

17 Ascospores striate-granularly ornamented, 8-10 $\times 3-4 \mu \mathrm{m}$. . Trichonectria leptogiicola Etayo

$17^{\prime}$ 'Ascospores smooth, 8.5-10.5 $\times 2.5-3 \mu \mathrm{m} \ldots \ldots \ldots \ldots$. . . . Nectriopsis collematis Diederich

18 Ascomatal wall $\mathrm{K}+$ violet, ascospores (19-)22-38(-65) $\times(7-) 9-11(-13) \mu \mathrm{m} \ldots \ldots \ldots . . . .$. . . . . . . . . . . . . . . . . . . . Xenonectriella lutescens (Arnold) Weese

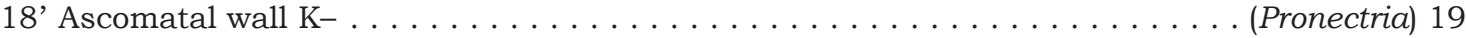

19 Ascomata with hyaline setae around the ostiole . . Pronectria pilosa Etayo \& López de Silanes

19' Ascomata without setae . . . . . . . . . . . . . . . . . . . . . . . . 20

20 Ascomata growing in the hymenium of the host, ascospores $5-7 \mu \mathrm{m}$ long. . . . . . . . .

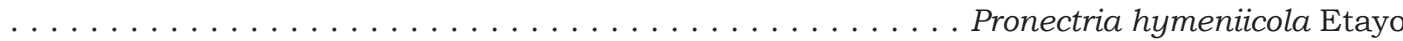

20 ' Ascomata growing on the thallus of the host, ascospores longer . . . . . . . . . . . 21

21 Ascospores easily disintegrating, secondarily developing $1-6$-celled units . . . . . . . . . . . . . . . . . . . . . . . . . . . . . . . Pronectria diplococca Kocourk. et al.

21 ' Ascospores remaining 1 -septate, not easily disintegrating . . . . . . . . . . . . 22

22 Ascospores smooth, 8.5-10.5 $\times 2.5-3 \mu \mathrm{m} \ldots$. . . . . . Pronectria collematis Etayo \& Brackel

22' Ascospores verruculose . . . . . . . . . . . . . . . . . . . . . . . 23

23 Ascomata yellowish, ascospores hyaline, c. $14-16.5 \times 5-6.5 \mu \mathrm{m} \ldots \ldots \ldots \ldots \ldots \ldots \ldots$

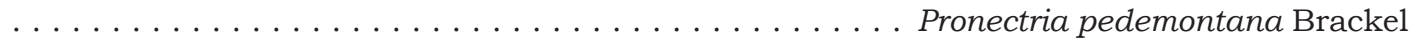

23" Ascomata red-orange, purple-red around the ostiole, ascospores pale orange, 10-16 × 5-6 $\mu \mathrm{m}$

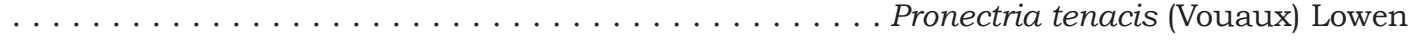




\section{REFERENCES}

Abbott, B. F. M. 2009. Checklist of the lichens and lichenicolous fungi of Greece. Bibliotheca Lichenologica 103: 1-368.

Aptroot, A., Diederich, P., Sérusiaux, E. \& Sipman, H. J. M. 1997. Lichens and lichenicolous fungi from New Guinea. Bibliotheca Lichenologica 64: 1-220.

Brackel, W. v. 2013. Miscellaneous records of lichenicolous fungi from the Italian Alps. Herzogia 26: 141-157. https://doi.org/10.13158/ heia.26.1.2013.141

Brackel, W. v. 2014. Kommentierter Katalog der flechtenbewohnenden Pilze Bayerns. Bibliotheca Lichenologica 109: 1-476.

Brackel, W. v. \& Berger, F. 2019. Lichenicolous fungi from Sardinia (Italy): new records and a first synopsis. Herzogia 32: 444-471. https://doi. org/10.13158/heia.32.2.2019.444

Brackel, W. v. \& Etayo, J. 2010. Pronectria collematis (Bionectriaceae, Hypocreales), a new species on Collema from Germany and Spain. Lichenologist 42: 361-364. https://doi.org/10.1017/ S0024282910000095

Calatayud, V., Navarro-Rosinés, P. \& Hafellner, J. 2002. A synopsis of Lichenostigma subgen. Lichenogramma (Arthoniales), with a key to the species. Mycological Research 106: 1230-1242. https://doi.org/10.1017/S095375620200655X

Calatayud, V., Navarro-Rosinés, P. \& Hafellner, J. 2013. Contributions to a revision of Cercidospora (Dothideales), 2: Species on Lecanora s.1., Rhizoplaca and Squamarina. Mycosphere 4: 539-557. https://doi.org/10.5943/mycosphere/4/3/8

Christensen, S. N. \& Alstrup, V. 2013. Notes on epilithic, epigeic and muscicolous lichens and lichenicolous fungi from rock autcrops in the mountains of northern Greece. Mycobiota 1: 25-50. https://doi. org/10.12664/mycobiota.2013.01.04

Clauzade, G., Diederich, P. \& Roux, C. 1989. Nelikenigintai fungoj likenlogaj. Ilustrita determinlibro. Bulletin de la Société Linnéenne de Provence, Numéro spéc. 1: 1-142.

David, J. C. \& Etayo, J. 1995. A new lichenicolous fungus from Collema: Endococcus caudisporus sp. nov. (Dothideales, incertae sedis). Lichenologist 27: 314-316. https://doi.org/10.1006/ lich.1995.0030

Diederich, P. \& Puntillo, D. 1995. New or interesting lichenicolous fungi: 7. Nectria brutia sp. nov. (Ascomycotina, Hypocreales). Bulletin de la Société des naturalistes luxembourgeois 96: 95-98.

Etayo, J. 2001. Hongos liquenícolas de Ecuador. I. Dos especies nuevas del orden Hypocreales (Ascomycota): Pronectria parmotrematis y Trichonectria leptogiicola. Anales del Jardín Botánico de Madrid 58(2): 219-222. https://doi.org/10.3989/ ajbm.2000.v58.i2.152

Etayo, J. 2002. Aportación al conocimiento de los hongos liquenicolas de Colombia. Bibliotheca Lichenologica 84: 1-154.
Etayo, J. 2010. Hongos liquénicolas de Peru. Bulletin de la Société Linnéenne de Provence 61: 83-128.

Etayo, J. \& Sancho, L. G. 2008. Hongos liquenícolas del Sur de Sudamérica, especialmente de Isla Navarino (Chile). Bibliotheca Lichenologica 98: 1-302.

Fleischhacker, A., Grube, M., Frisch, A., Obermayer, W. \& Hafellner, J. 2016. Arthonia parietinaria-a common but frequently misunderstood lichenicolous fungus on species of the Xanthoria parietinagroup. Fungal Biology 120: 1341-1353. https:// doi.org/10.1016/j.funbio.2016.06.009

Grube, M. \& Hafellner, J. 1990. Studien an flechtenbewohnenden Pilzen der Sammelgattung Didymella (Ascomycetes, Dothideales). Nova Hedwigia 51: 283-360.

Hafellner, J. 2009. Phacothecium resurrected and the new genus Phacographa (Arthoniales) proposed. Bibliotheca Lichenologica 100: 85-121.

Hawksworth, D. L. 1980. Notes on British lichenicolous fungi III. Notes from the Royal Botanical Garden Edinburgh 38: 165-183.

Hawksworth, D. L. 1981. The lichenicolous coelomycetes. Bulletin of the British Museum, Botany 9: 1-98.

Hoffmann, N. \& Hafellner, J. 2000. Eine Revision der lichenicolen Arten der Sammelgattungen Guignardia und Physalospora (Ascomycotina). Bibliotheca Lichenologica 77: 1-181.

Keissler, K. v. 1930. Die Flechtenparasiten. - In: Dr. L. Rabenhorst's Kryptogamen-Flora von Deutschland, Österreich und der Schweiz. 2. Aufl., Bd. 8., Leipzig.

Khodosovtsev, A., Vondrák, J., Naumovich, A., Kocourková, J., Vondráková, O. \& Motiejūnaitè, J. 2012. Three new Pronectria species in terricolous and saxicolous microlichen communities (Bionectriaceae, Ascomycota). Nova Hedwigia 95: 211-220. https://doi.org/10.1127/00295035/2012/0026

López de Silanes, M. E., Etayo, J. \& Paz-Bermúdez, G. 2009. Pronectria pilosa (Hypocreaceae) sp. nov. and other lichenicolous fungi found on Collemataceae in the Iberian Peninsula. Bryologist 112: 101-108. https://doi.org/10.1639/00072745-112.1.101

Navarro-Rosinés, P., Etayo, J. \& Calatayud, P. 1999. Rhagadostoma collematum sp. nov. (ascomicetes liquenícolas, Sordariales) y nuevos datos para otras especies del género. Bulletin de la Société Linnéenne de Provence 50: 233-241.

Navarro-Rosinés, P. \& Hladún, N. L. 1990. El género Sarcopyrenia Nyl. (ascomicetes liquenícolas) en Europa y norte de Africa. Candollea 45: 469-489.

Nylander, W. 1873. Observata lichenologica in Pyrenaeis orientalibus. Flora 56: 194-207.

Orange, A. 1990. New or interesting lichens and lichenicolous fungi from Iceland. Acta Botanica Islandica 10: 37-44.

Rossman, A. Y., Samuels, G. J., Rogerson, C. T. \& Lowen, R. 1999. Genera of Bionectriaceae, Hypo- 
creaceae and Nectriaceae (Hypocreales, Ascomycetes). Studies in Mycology 42: 1-248.

Roux, C. \& Triebel, D. 1994. Révision des espèces de Stigmidium et de Sphaerellothecium (champignons lichénicoles non lichénisés, Ascomycetes) correspondant à Pharcidia epicymatia sensu Keissler ou à Stigmidium schaereri auct. Bulletin de la Société Linnéenne de Provence 45: 451-542.

Roux, C., Triebel, D., Bricaud, O. \& Le Coeur, D. 1995. Le Stigmidium lecidellae sp. nov. et remarques sur le genere Stigmidium (champignons lichénicoles non lichénisés, Ascomycetes). Canadian Journal of Botany 73: 662-672. https://doi.org/10.1139/ b95-070

Sipman, H. J. M. \& Raus, T. 2015. Lichens and lichenicolous fungi from the island of Chios (Aegean Sea, Greece). Herzogia 28: 496-519. https://doi. org/10.13158/heia.28.2.2015.496

Timdal, E. 1991. A monograph of the genus Toninia (Lecideaceae, Ascomycetes). Opera Botanica 110: $1-137$.

Vondrák, J., Guttova, A. \& Mayrhofer, H. 2008. A further contribution to the knowledge of lichen- forming and lichenicolous fungi in Crete. Herzogia 21: 105-124.

Vouaux, A. 1913. Synopsis des champignons parasites des lichens. Bulletin de la Société Mycologique de France 29: 33-128, 399-446, 447-494.

Vouaux, A. 1914. Synopsis des champignons parasites des lichens. Bulletin de la Société Mycologique de France 30: 135-198, 281-329.

Wagner, H.-G. \& Schacherer, A. 2019. Einige für Niedersachsen neue lichenicole Pilze sowie weitere bemerkenswerte Funde. Braunschweiger Naturkundliche Schriften 15: 45-79.

Winter, G. 1872. Diagnosen und Notitzen zu Rehm's Ascomyceten. Flora 55: 508-511, 523-527, 542-544.

Zamora, J. C., Diederich, P., Milanes, A. M. \& Wedin, M. 2017. An old familiar face: Tremella anaptychiae sp. nov. (Tremellales, Basidiomycota). Phytotaxa 307: 254-262. https://doi.org/10.11646/ phytotaxa.307.4.3

Zhurbenko, M. P. 2009. New and interesting lichenicolous hypocrealean fungi from the Northern Hemisphere. Sydowia 61: 177-188. 